
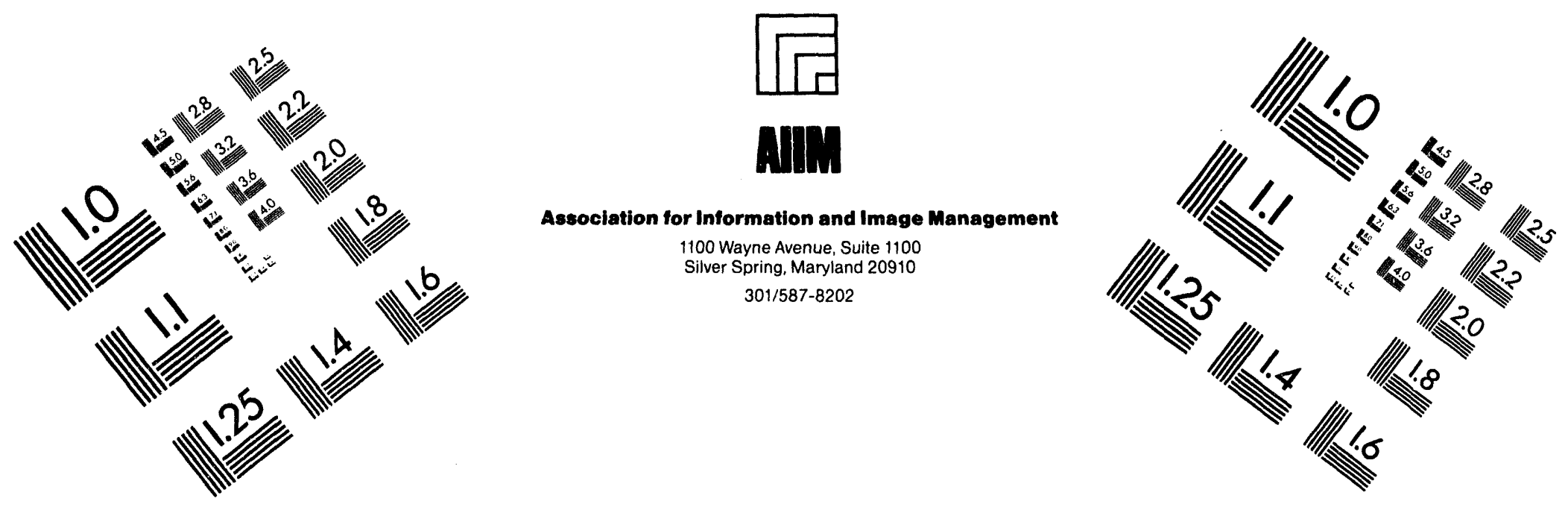

\title{
Centimeter
}

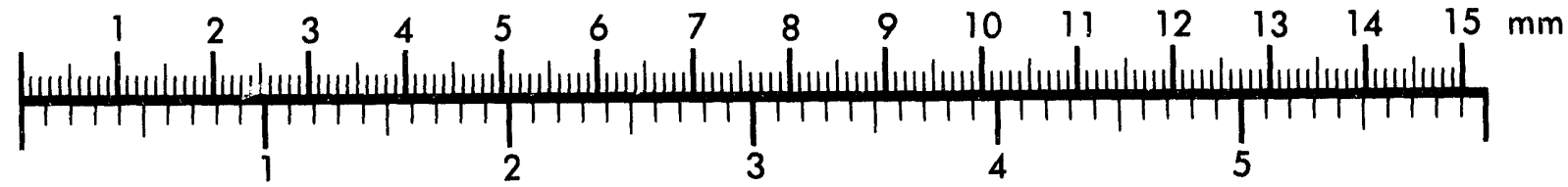
Inches
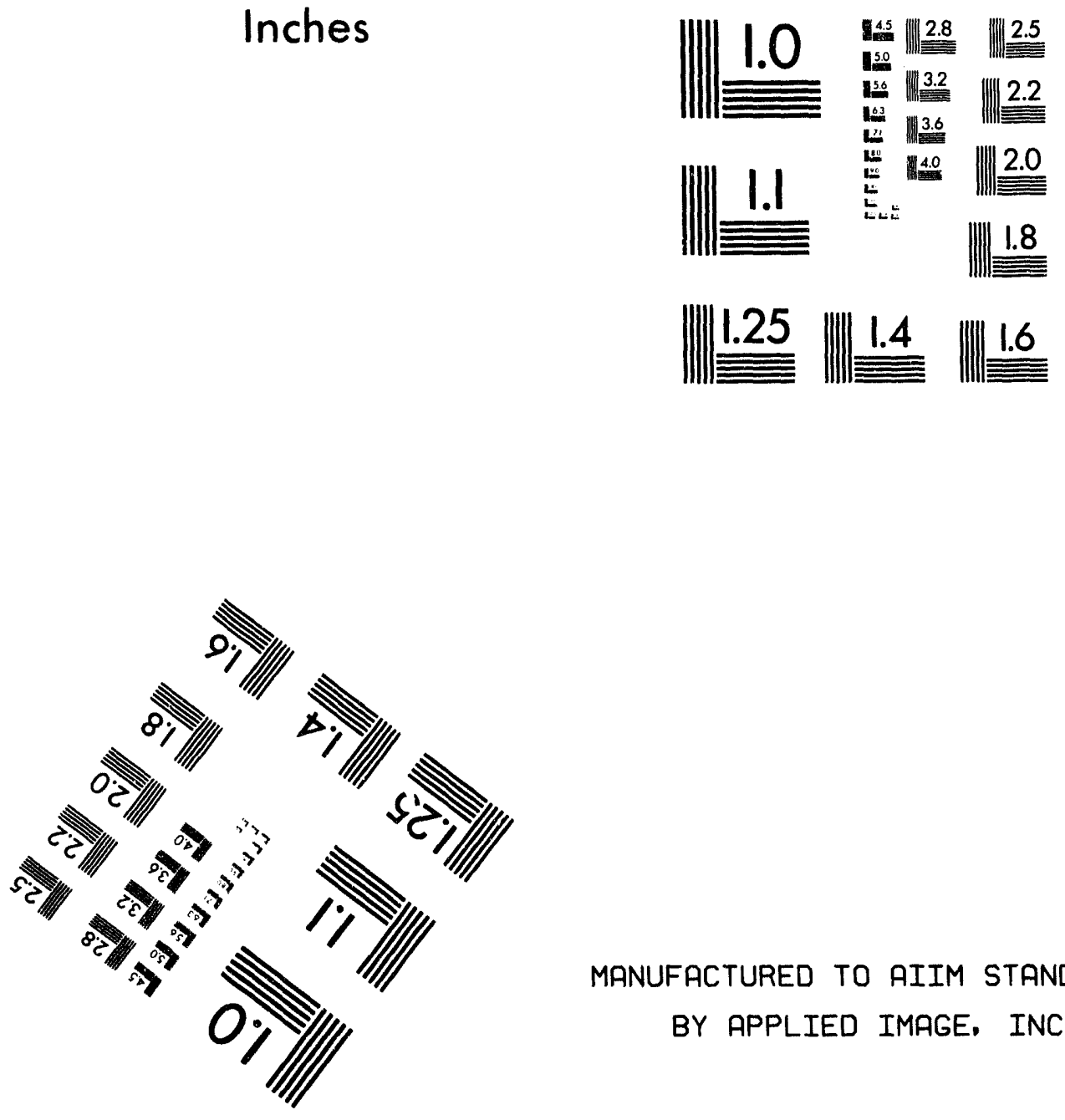

MANUFACTURED TO AIIM STANDARDS

BY APPLIED IMAGE, INC.

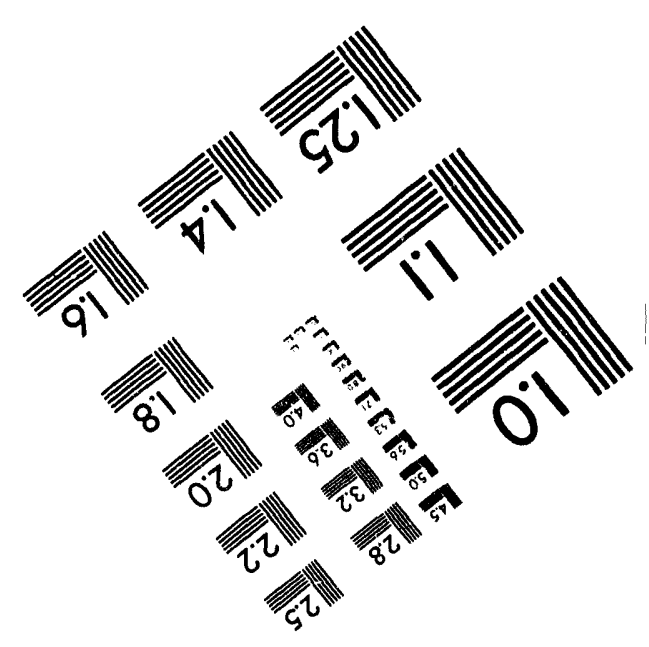



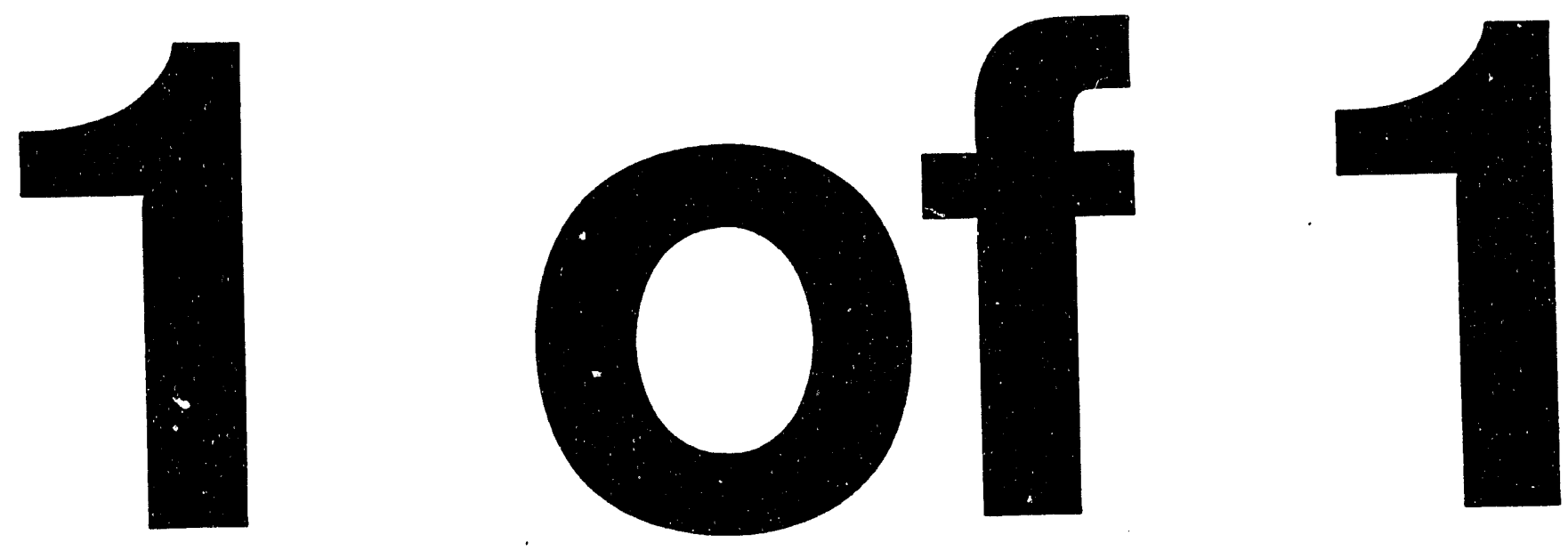


\title{
CONF-931108-104
}

\author{
Proceedings of \\ Fall Meeting of the Materials Research Society \\ Boston, Massachusetts \\ November 29-December 3, 1993
}

\section{SIMULATIONS OF THE DISLOCATION ARRAY AT Ge/Si INTERFACES}

Theodore Kaplan, M. F. Chisholm, and Mark Mostoller

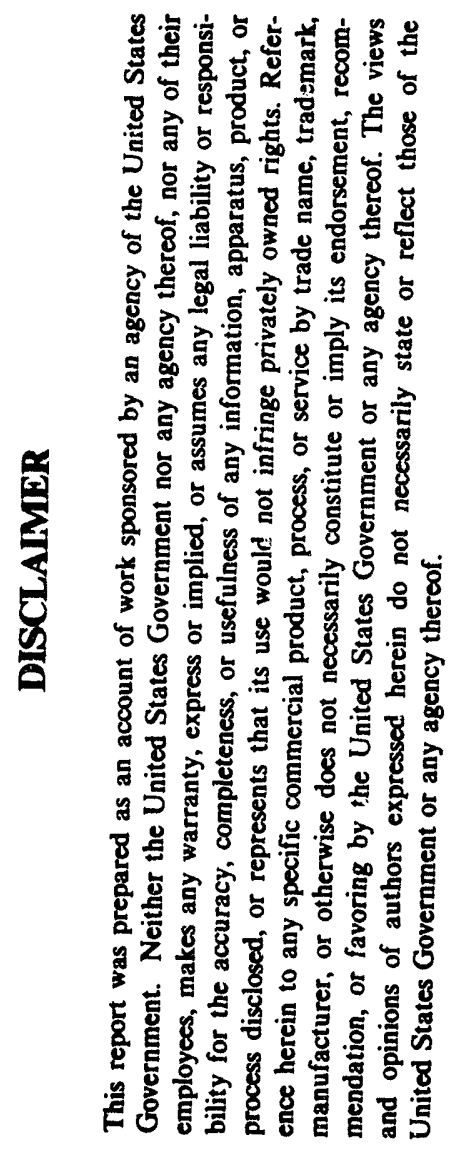

"The submitted manuscript has been authored
by contractor of the U.S. Govemment under
contract No. DE.AC05-840R21400.
Accordingly, the U.S. Government retains
nonexclusive, royalty-free license to publish or
reproduce the published form of this
contribution, or allow others to do so, for U.S.
Government purposes."

\author{
SOLID STATE DIVISION \\ OAK RIDGE NATIONAL LABORATORY \\ Managed by \\ MARTIN MARIETTA ENERGY SYSTEMS, INC. \\ Under \\ Contract No. DE-AC05-84OR21400 \\ With the \\ U. S. DEPARTMENT OF ENERGY \\ OAK RIDGE, TENNESSEE
}

December 1993

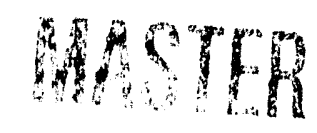




\title{
SIMULATIONS OF THE DISLOCATION ARRAY AT Ge/Si INTERFACES
}

\author{
THEODORE KAPLAN, M. F. CHISHOLM, AND MARK MOSTOLLER
}

Solid State Division, Oak Ridge National Laboratory. Oak Ridge, TN 37831

\begin{abstract}
When Ge is grown epitaxially on $\mathrm{Si}(001)$, the $4 \%$ mismatch between the lattice parameters of $\mathrm{Ge}$ and $\mathrm{Si}$ can produce a regular two-dimensional grid of $(a / 2)[1, \pm 1,0]$ edge dislocations at the interface, a checkerboard with a spacing of $\sim 100 \AA$. We have performed classical molecular dynamical simulations of this checkerboard in large microcrystals. The results show the expected 5-fold plus 7-fold ring structure at the cores of the individual dislocations, and a new closed symmetric structure of 18 atoms at their intersections. Tetrahedral coordination is everywhere retained, with relatively small changes in the bond lengths of less than $10 \%$ and in the bond angles of less than $25 \%$. The energetics and dislocation offset of the system are explored for the Stillinger-Weber and Tersoff potentials.
\end{abstract}

\section{DISCUSSION AND RESULTS}

There is a $4 \%$ lattice mismatch between silicon and germanium $(a=5.43,5.65 \AA$ respectively). When $\mathrm{Ge}$ is grown epitaxially on an $\mathrm{Si}(001)$ substrate, the mismatch strain can be relieved by formation of a square array of edge dislocations with Burgers' vectors $(a / 2)[1, \pm 1,0]$ parallel to the interface plane. 1 The spacing in this array is 24 layers of Ge or 25 layers of $\mathrm{Si}$ along the orthogonal $<1,1,0\rangle$ directions, which is $96 \AA$. Of particular interest to us was if and how the dislocations might intersect. Such intersections would have a planar density of $\sim 10^{12} / \mathrm{cm}^{2}$, certainly large enough to have an impact on the electrical properties of the interface and in a Ge film some tens of layers thick.

Single $(a / 2)<1,1,0>$ edge dislocations in $\mathrm{Si}$ or Ge have a core structure of 5-fold and 7-fold rings stacked along the (001) direction, as illustrated in Fig. 1. This structure was first

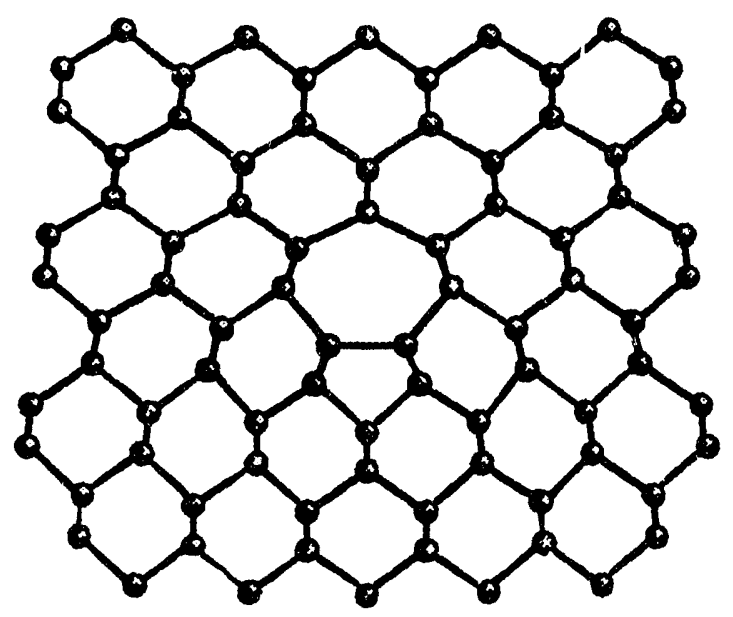

Fig. 1. The 5-fold plus 7 -fold ring structure at the core of the $(a / 2)[1,1,0]$ edge dislocation in $\mathrm{Si}$.

predicted by Hornstra in 1957,2 and more recently, Nandedkar and Narayan ${ }^{3}$ have done classical simulations that show that it occurs. Current understanding of dislocations cores in Si has been reviewed by Markland ${ }^{4}$ and by Duesbery and Richardson. ${ }^{5}$ Each atom in the core retains tetrahedral bonding. The same linked 5-plus 7-fold structure has been predicted in several other circumstances, including the $90^{\circ}$ partial in $\mathrm{Si}^{6}$ the $\Sigma 9$ twin boundary, 7,8 and the $\mathrm{Si}(111): 2 \times 1$ 
surface. 9 Changes in bond lengths of the order of 5\% and in bond angles of as much as 25-30\% have been reported in these studies.

To determine the structure that might be found for the dislocation grid at the interface of $\mathrm{Ge} / \mathrm{Si}$ bicrystals, we have performeu classical molecular dynamics simulations in samples of roughly 20,000 atoms. The potential models employed were those of Stillinger and Weber (SW) $10-12$ and of Tersoff. ${ }^{13}$ These have been used in the literature for a wide variety of problems. Each in its own fashion favors the diamond cubic structure by adding three-body angular forces that penalize deviations from tetrahedral bonding. While these potential models have enjoyed some success (see Ref. 6 for the $90^{\circ}$ partial, for example), they also have some shortcomings which we will discuss later on. One shortcoming will be taken up immediately, however, because it played a role in the way the simulations were done.

With the present generation of computers, we cannot simulate the actual growth of a film of $\mathrm{Ge}$ on $\mathrm{Si}$ and the formation of a grid of dislocations spaced $100 \AA$ apart. For single edge dislocations, however, the equilibrium structure can be found by the textbook procedure of removing a half-plane and minimizing the total energy; that is how Fig. 1 was obtained. A procedure like this for the Ge/Si bicrystal would be simply to stack the Ge film on top of the $\mathrm{Si}$ substrate and minimize. Unfortunately, this does not work for two reasons. First, there are extremely large strains and forces at the interface in the starting sample. Second, both the SW and Tersoff potentials have very short range, with the pair potential contributions cut off inside the 2 nd nearest neighbor $(2 \mathrm{NN})$ distance. This means first that non-equilibrium structures will tend to form in the initial steps of minimization, and second, that these structures will tend to be locked in.

Several alternative procedures were tried, and the following summarizes the series of steps finally taken. Si and Ge half crystals with orthogonal axes along $x=[1,-1,0] / \sqrt{2}, y=[1,1,0] / \sqrt{2}$, and $z=[0,0,1]$ were constructed and stacked, and the sample was then prehealed to somewhat reduce the large strains and forces at the interface and to predispose the sample to form $(a / 2)[1, \pm 1,0]$ edge dislocations intersecting near the middle. Periodic boundary conditions were applied along the $\mathrm{x}$ and $\mathrm{y}$ directions, and free surfaces along $\mathrm{z}$. Because the potentials have such short range, simulated annealing from high temperature (T) produced and locked in an amorphous structure at the interface. To deal better with the very large forces in the starting sample, we instead began with a number of time steps at very low $\mathrm{T}\left(\mathrm{a}\right.$ few $\left.{ }^{\circ} \mathrm{K}\right)$, rescaling the velocities every time step to this low $\mathrm{T}$ value to suck out energy. This was followed by a series of simulated annealing runs from high $\mathrm{T}(\sim 600 \mathrm{~K})$ to low, with a final run using the conjugate gradient method to minimize the total potential energy.

The structural results for the orthogonal dislocations in the grid are the same as those for isolated edge dislocations, the stacked 5- plus 7-fold rings shown in Fig. 1. This was not unexpected, but did provide some level of confidence in the simulations. In the grid, the dislocation cores along $\mathrm{x}$ and $\mathrm{y}$ are displaced vertically relative to each other by a single layer $a / 4$ along $[0,0,1]$. Intersections therefore do occur, and the result is the closed symmetric structure of 18 atoms shown in Fig. 2. It contains four 5-fold rings at the bottom and four 7-fold rings at the top, joined to form an extended point defect with the $2 \mathrm{~mm}$ point group. For brevity, we will call this intersection the dreidl, after the child's top that it evokes. 14

We have done a number of exploratory studies of the structure, energetics and electronic structure of this new extended point defect predicted in diamond cubic and zincblende crystals. It may occur, like the simpler 5-plus 7 -fold ring structure of single edge dislocations, in other situations like grain boundaries or domain walls. Because our results are preliminary in nature, we will summarize them briefly.

Tetrahedral coordination is retained by the 18 atoms in the dreidl, but the changes in bond lengths and angles are somewhat larger than for single dislocations, as are the strain energies. Bond length changes are as much as $9-10 \%$ in our calculations (cf. $5-8 \%$ for the cores of the individual dislocations in the grid 4-5 layers away from their intersection, depending on the potential model), and angles change from their tetrahedral values by up to $25 \%(20 \%)$. Strain energies in the core of the dreidl appear to be roughly additive, reaching $10 \%$ or about $0.4 \mathrm{eV}$ per atom $(0.2 \mathrm{eV})$. 


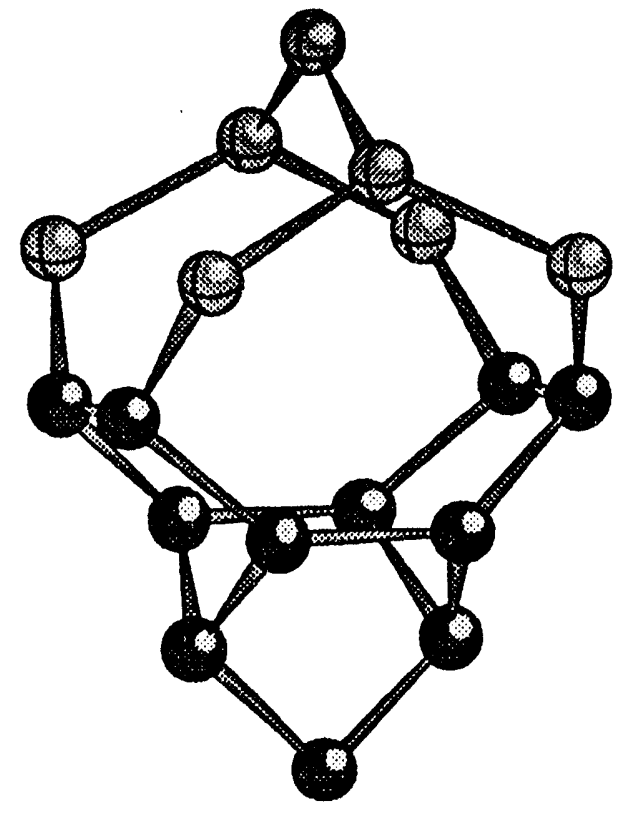

Fig. 2 The dreidl, the closed symmetric structure of 18 atoms predicted at the intersections of the dislocation grid in $\mathrm{Ge} / \mathrm{Si}$ bicrystals. The $[0,0,1]$ direction is vertical in the figure.

These larger strains may presage the possibility that the dreidl is electrically active. We have tried to explore this by constructing a much smaller sample that can be periodically repeated in all three dimensions in order to do electronic structure calculations. This sample was built by taking the relaxed structure of $\sim 20,000$ atoms and slimming it along $z=[0,0,1]$ from 16 layers each of $\mathrm{Si}$ and $\mathrm{Ge}$ to one of $6+6$ and a second of 5+3 layers, rotating the second by $90^{\circ}$ around $\mathrm{z}$, inverting it and stacking it on top of the first, and cropping to 12 and 14 layers along $x$ and $y$ in $\mathrm{Ge}$ and $\mathrm{Si}$ along 6-fold rings. This perhaps confusing outline of the procedure produces an 849 atom microcrystal that is shown in Fig. 3 periodically repeated along $x=[1,-1,0]$. Careful

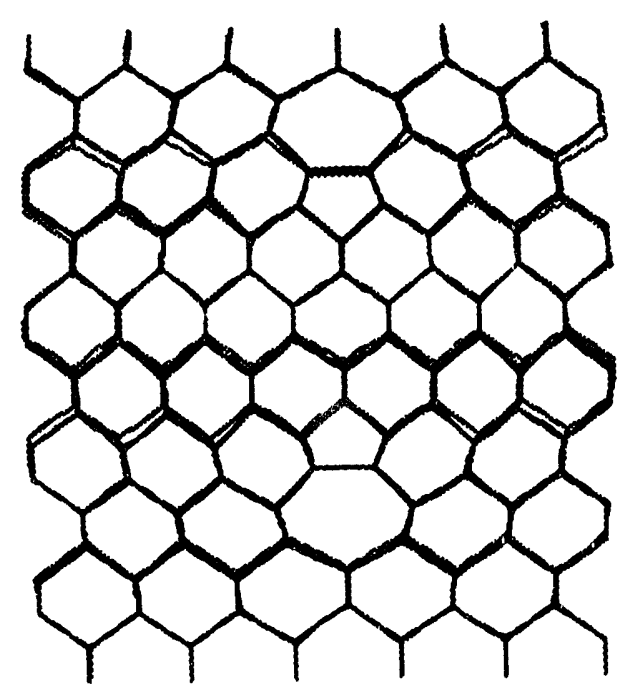

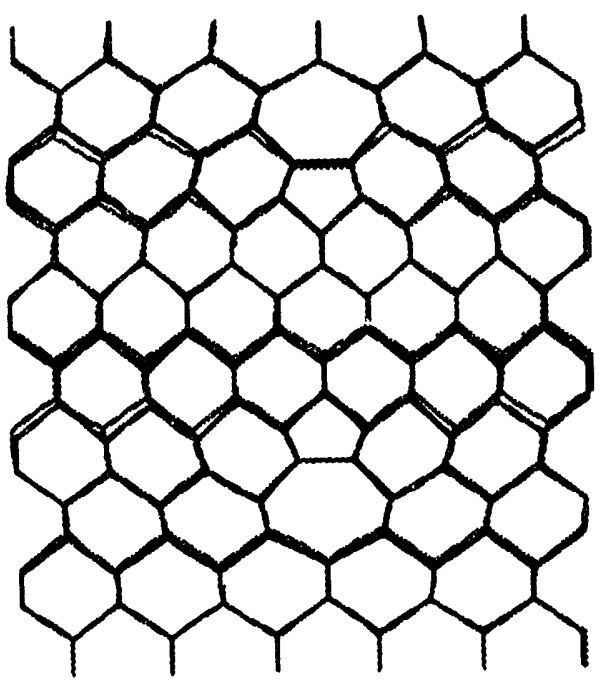

Fig. 3. A stack of dislocation grids and their intersections constructed to allow periodic repetition in three dimensions. The structure is shown repeated along $x=[1,-1,0] / \sqrt{2}$.

inspection of the figure will demonstrate that the 849-atom unit cell can in fact be periodically repeated along $z$ as well as $x$, and a similar picture obtains for the $y$ direction. The atomic positions in the $x$ and $y$ directions have so far been kept fixed at the values found in the 
simulations for the much larger system, so considerable strain remains across the boundaries of the smaller sample when it is periodically repeated (see Fig. 3).

Preliminary tight-binding calculations have been done for this unrelaxed 849 atom periodic structure using the s-p ${ }^{3}-\mathrm{s}^{*}$ approach of Vogl et. al. 15 These show a split-off band of states in the gap extending several tenths of volts above the valence band. This is in contrast to the results of the LDA calculations for the $90^{\circ}$ partial, ${ }^{6}$ where smaller changes in bond lengths and angles produced only very shallow states in the gap. However, we cannot yet conclusively identify the states in the split-off band as localized at the dreid! rather than, for example, at the strained regions in the boundaries of this periodically repeated sample.

With the classical potential models, we have done some rough studies of the energetics of the location of the dislocation grid relative to the Ge-Si interface. This is a problem for which both the SW and Tersoff potentials are ill-suited, because neither does an adequate job of treating the long-range strain fields of dislocations. If isotropic linear elasticity theory is used to describe these long-range strains and energies, a combination $\mathrm{K}$ of the elastic constants governs, ${ }^{16}$

$$
\begin{gathered}
K=\left(C_{12}+c_{12}\right)\left\{c_{44}\left(C_{12}-c_{12}\right) /\left[c_{11}\left(C_{12}+c_{12}+2 c_{44}\right)\right]\right\}^{1 / 2} \\
C_{12}=\left[c_{11}\left(c_{11}+c_{12}+2 c_{44}\right)\right]^{1 / 2}
\end{gathered}
$$

Experimentally, the values of $\mathrm{K}$ are 0.904 for $\mathrm{Si}$ and 0.733 for $\mathrm{Ge}$, favoring the formation of the dislocations in the $\mathrm{Ge}$ film as has been observed. ${ }^{1}$ For the potential models used here, however, there are essentially no differences in $K$ for the two materials: $S W-K=0.710,0.704 \times 10^{12}$ dyn $/ \mathrm{cm}^{2}$ for $\mathrm{Si}, \mathrm{Ge}$, respectively; Tersoff $-\mathrm{K}=0.775,0.754$.

We have done simulations in which we moved the dislocation grid and dreidl up and down so that they are wholly in the Ge film or in the Si substrate rather than straddling the interface as in Figs. 1 and 2. This was done by the expedient of changing $\mathrm{Si}$ atoms to $\mathrm{Ge}$ or vice versa. The results were assessed on the basis of strain energy per atom for the 10 planes in $\mathrm{Si}$ and 10 planes in $\mathrm{Ge}$ closest to the interface, thereby including at least three planes above and below the grid. The number of atoms involved in these averages is about 12,000, and it is not quite constant because we are changing larger numbers of $\mathrm{Si}$ atoms per plane to $\mathrm{Ge}$ or the reverse but keeping a constant number of Ge layers and of Si layers to compute the average strain energy per atom. The results are summarized in Table I. As might be expected from the values that the

Table I. Calculated strain energies (SE) for the ten planes each of $\mathrm{Ge}$ and Si closest to the interface with the dislocation grid straddling the interface, wholly in Ge, and wholly in Si.

\begin{tabular}{lllll}
\hline Location & \# Ge & $\# \mathrm{Si}$ & Total & SE/atom (\%) \\
& & & & \\
Interface & 5760 & 6225 & 11985 & 0.324 \\
$\mathrm{Ge}$ & 5931 & 6250 & 12181 & 0.428 \\
$\mathrm{Si}$ & 5760 & 6078 & 11838 & 0.412 \\
\hline
\end{tabular}

potential models give for $\mathrm{K}$, there is little preference indicated as to location, but a slight advantage is shown for the grid straddling the interface. We believe this is probably due to the shortcomings of the potential models, and are working to improve them.

In summary, we have predicted a new defect structure in diamond cubic semiconductors. The dreidl is a large point defect structure that may occur in high planar densities at $\mathrm{Ge} / \mathrm{Si}$ interfaces as well as in other boundaries, and that may be electrically active. Further experimental and theoretical studies are in progress. 


\section{ACKNOWLEDGMENTS}

We are grateful to Benjamin Liu, L. J. Gray, and Mark Robinson for helpful conversations. Victor Milman and Feng Liu have been gracious in doing exploratory calculations of electronic structure. This research was sponsored by the Division of Materials Sciences, U.S. Department of Energy under Contract No. DE-AC05-84OR21400 with Martin Marietta Energy Systems, Inc.

\section{REFERENCES}

1. M. F. Chisholm, S. J. Pennycook, and D. E. Jesson, Mat. Res. Soc. Symp. Proc. 158, 447 (190).

2. J. Hornstra, J. Phys. Chem Solids 5, 129 (1958).

3. A. S. Nandedkar and J. Narayan, Phil. Mag. A 56, 625 (1987); Phil. Mag. A 61, 873 (1990).

4. S. Markland, J. Phys. C 4, 44 (1983).

5. M. S. Duesbery and G. Y. Richardson, Solid State and Materials Sciences 17, 1 (1991).

6. J. R. K. Bigger, D. A. McInnes, A. P. Sutton, M. C. Payne, I. Stich, R. D. King-Smith, D. M. Bird, and L. J. Clarke, Phys. Rev. Lett. 69, 2224 (1992).

7. R. E. Thomson and D. J. Chadi, Phys. Rev. B 29, 889 (1984).

8. D. P. DiVincenzo, O. L. Alerhand, M. Schluter, and J. W. Wilkins, Phys. Rev. Lett. 56, 1925 (1986).

9. K. C. Pandey, Phys. Rev. Lett. 47, 1913 (1981).

10. F. H. Stillinger and T. A. Weber, Phys. Rev. B 31, 5262 (1985).

11. K. Ding and C. Andersen, Phys. Rev. B 34, 6987 (1987).

12. M. Karimi, T. Kaplan, M. Mostoller, and D. E. Jesson, Phys. Rev. B 47, 9931 (1993).

13. J. Tersoff, Phys. Rev. B 39, 5566 (1989).

14. dreidl trhymes with cradlel 1: a 4-sided toy marked with Hebrew letters and spun like a top in a game of chance, Webster's Ninth New Collegiate Dictionary. (Merriam-Webster, Springfield, 1989)

15. P. Vogl, H. P. Hjalmarson. and J. D. Dow, J. Phys. Chem. Solids 44, 365 (1983).

16. A. J. E. Foreman, Acta Met. 3, 322 (1955). 

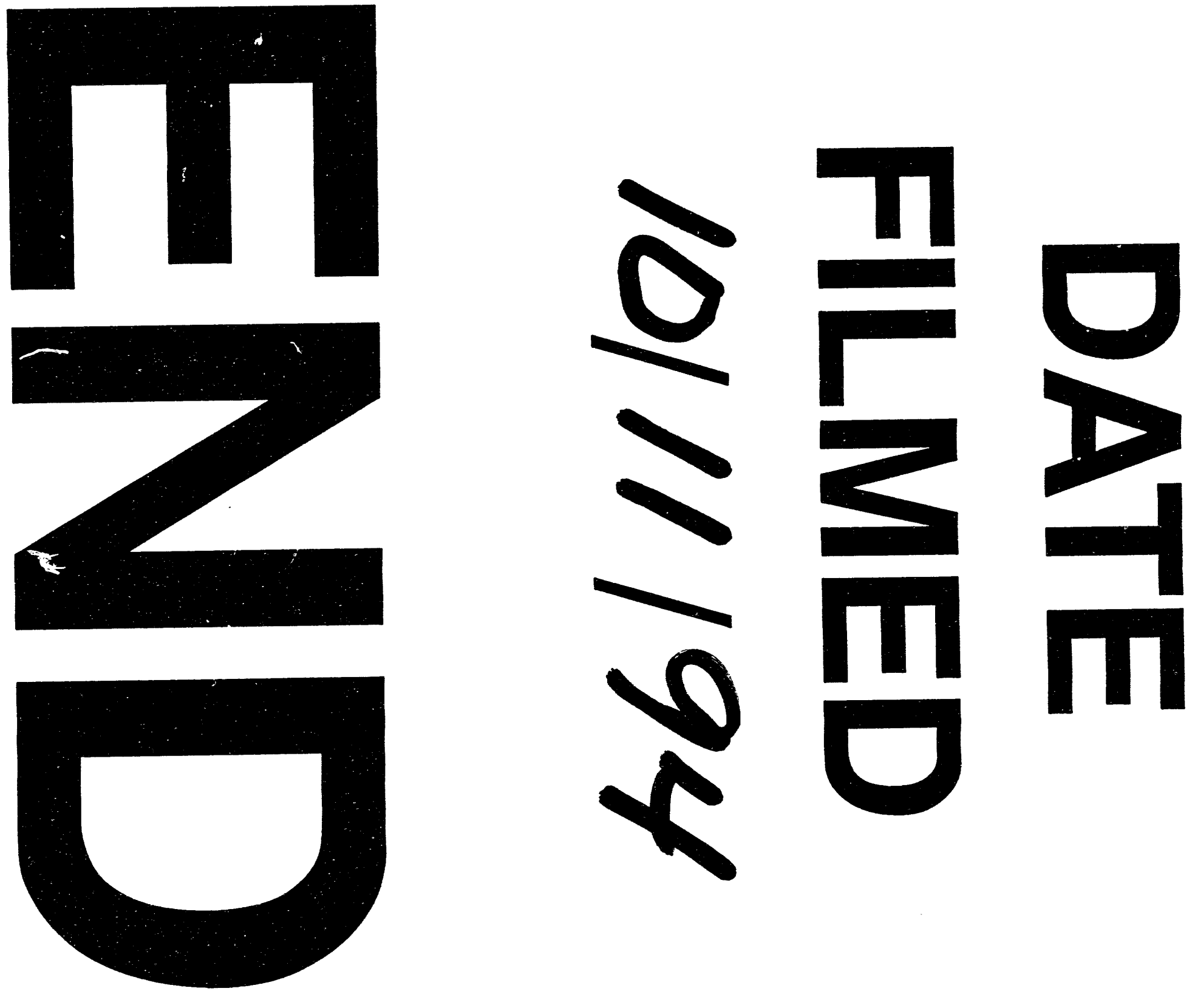


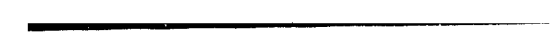

